\title{
Evaluation of a Family-Based Group Therapy for Young Chil- dren with Obesity: A Pilot Study
}

\begin{abstract}
Albane Bertha Rosa Maggio ${ }^{1 *}$, Michelle Mugnier ${ }^{1}$, Lily Piaget ${ }^{1}$, Maude Bessat-Macchi ${ }^{1,2}$, Xavier Martin ${ }^{1}$, Nathalie FarpourLambert $^{1,2}$, Virginie Desmangles ${ }^{3}$, Anne Romanowicz ${ }^{3}$, Jean-Sébastien Landry ${ }^{3}$
\end{abstract}

${ }^{1}$ Department of Child and Adolescent, University of Geneva, Switzerland

${ }^{2}$ Department of Community Medicine, University of Geneva, Switzerland

${ }^{3}$ Geneva Pediatric Network, Switzerland

"Corresponding author: Albane Bertha Rosa Maggio, Department of Child and Adolescent, University of Geneva, Switzerland Tel: +41223823311; Fax +41223725090; Email: albane.maggio@hcuge.ch

Citation: Maggio ABR, Mugnier M, Piaget L, Bessat-Macchi M, Martin X, et al. (2017) Evaluation of a Family-Based Group Therapy for Young Children with Obesity: A Pilot Study. J Family Med Prim Care Open Acc: JFOA-102. DOI: 10.29011/JFOA102. 100002

Received Date: 6 October, 2017; Accepted Date: 30 October, 2017; Published Date: 6 November, 2017

\begin{abstract}
Obesity management should be initiated early during childhood. We aimed to assess the effects and factors influencing an intervention combining physical activity and Family-Based Behavioral Treatment (FBBT) in group setting, on Body Mass Index (BMI) and psychological co-morbidities in 3 to 7 years old children with obesity. This is a clinical trial pilot study including 17 overweight or obese children, aged 3 to 7 years old, and their parents. The low-intensity intervention included 9 group sessions based on the FBBT approach spread over 12 months. For the 13 subjects who completed the study $(76.5 \%)$, the BMI z-score was stable during the first 6 months (delta BMI z-score: $0.06 \pm 0.3$ ), but increased at one year $(0.23 \pm 0.4)$. At 12 months, it was influenced by the psychological states of the child and father (child emotional problems: $r=0.606, p=0.048$; father depression: $r=0.821, p=0.012$; father anxiety: $r=0.723, p=0.043$ ). Conclusions: This pilot study suggests that there is a relationship between BMI z-score in young children and children behavior, father support, and psychological disorders. This is an interesting issue, with novel results.
\end{abstract}

Keywords: Children; Family; Obesity; Psychological States; Weight Management Program

\section{Abbreviations:}

FBBT : $\quad$ Family-Based Behavioral Treatment

BMI : $\quad$ Body Mass Index

SDQ : $\quad$ Strengths and Difficulties Questionnaire

BDI : $\quad$ Beck Depression Inventory

BAI : Beck Anxiety Inventory

\section{What Is Known:}

- Family patterns of eating behaviors have a role in the child's weight.
- There is a higher frequency of depressive disorders in parents of obese children and adolescents compared with parents of non-obese children.

\section{What Is New:}

- The father's support and psychological state has a crucial role to maintain long term participation to a weight management program.

\section{Introduction}

The Family-Based Behavioral Treatment (FBBT) was developed to modify the shared family environment, and to provide role models and support for child behavior changes [1]. We aimed to assess the effects of a low-intensity intervention combining physical activity and FBBT in group setting, on Body Mass Index 


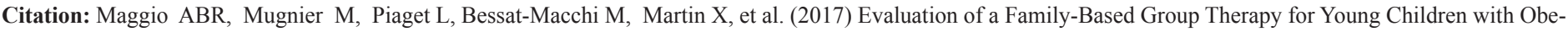
sity: A Pilot Study. J Family Med Prim Care Open Acc: JFOA-102. DOI: 10.29011/JFOA-102. 100002

(BMI) and psychological co-morbidities in 3 to 7 years old children with obesity and their parents. We also aimed to investigate factors influencing treatment adherence and success.

\section{Methods}

This was a clinical trial pilot study including 13 overweight or obese children, aged 3 to 7 years old, and their parents. The « Ethics Commission for Research on the Human Being » (CEREH) ( $n^{\circ}$ de ref. CER :13-172) ethics committee approved this study and a written informed consent was obtained from parents. The intervention included 9 one-hour group sessions spread over 12 months. Parents and children sessions were held separately. The parents' sessions were co-led by two health care professionals: one dietitian and one psychologist. Children sessions were directed by a psychomotor therapist, a pediatrician and a nurse.
We assessed anthropometrics variables at baseline, after 6 and 12 months using standardized methods. The French version of the Strengths and Difficulties Questionnaire (SDQ)[2], the French versions of the Beck Depression Inventory (BDI) [3] and the Beck Anxiety Inventory (BAI) [4] were filled by parents at baseline and after 12 months. Statistical analyses were performed using the SPSS software 18.0 (Chicago, IL). Pearson coefficient correlation, paired t-test, independent Student's t-test and Chi-2 were used when appropriate. BMI z-score was considered stable if the change was \pm 0.1 . Differences were considered significant if $\mathrm{p}<0.05$.

\section{Results}

Characteristics of the subjects and SDQ questionnaire results are presented in (Table 1). Results of the BDI and the BAI are presented in (Table 2). The BMI z-score was stable at 6 months, but there was a trend towards an increase at 12 months (Table 1).

\begin{tabular}{|c|c|c|c|c|}
\hline & $\underbrace{\text { All }}_{\mathrm{N}=13}$ & $\begin{array}{c}\text { Low attendance rate } \\
(<75 \%) \\
\mathrm{N}=\mathbf{6}\end{array}$ & $\begin{array}{c}\text { High attendance rate } \\
(>75 \%) \\
\mathrm{N}=7\end{array}$ & $\underline{\mathbf{p}}$ \\
\hline & Mean \pm SD & Mean \pm SD & Mean \pm SD & \\
\hline Mean participation rate, $\%$ & $75.2 \pm 20.9$ & $57.4 \pm 13.0$ & $90.5 \pm 11.9$ & 0.001 \\
\hline \multicolumn{5}{|c|}{ Characteristics } \\
\hline Age, years & $6.0 \pm 1.1$ & $6.2 \pm 1.4$ & $5.9 \pm 1.0$ & NS \\
\hline BMI z-score at baseline, $\mathrm{kg} \cdot \mathrm{m}^{-2}$ & $2.8 \pm 0.9$ & $2.9 \pm 1.0$ & $2.8 \pm 0.8$ & NS \\
\hline Delta BMI zs at 6 months, kg.m ${ }^{-2}$ & $0.06 \pm 0.3$ & $0.03 \pm 0.2$ & $0.09 \pm 0.3$ & NS \\
\hline Delta BMI zs at 12 months, kg.m ${ }^{-2}$ & $0.23 \pm 0.4$ & $0.21 \pm 0.4$ & $0.25 \pm 0.5$ & NS \\
\hline \multicolumn{5}{|c|}{ Psychological factors } \\
\hline \multicolumn{5}{|l|}{ SDQ questionnaire } \\
\hline Pro-social behavior & $7.6 \pm 3.9$ & $9.0 \pm 1.2$ & $6.7 \pm 2.4$ & NS \\
\hline Emotional problems & $3.5 \pm 2.2$ & $4.0 \pm 2.9$ & $3.1 \pm 1.8$ & NS \\
\hline Conduct problems & $2.9 \pm 2.2$ & $2.8 \pm 2.2$ & $3.0 \pm 2.4$ & NS \\
\hline Hyperactivity/inattention & $2.9 \pm 1.7$ & $3.3 \pm 2.1$ & $2.7 \pm 1.6$ & NS \\
\hline Peer relationship problems & $1.9 \pm 1.5$ & $1.7 \pm 1.2$ & $2.0 \pm 1.6$ & NS \\
\hline Total difficulty score & $11.6 \pm 3.9$ & $13.3 \pm 0.6$ & $10.9 \pm 4.6$ & NS \\
\hline
\end{tabular}

Table 1: Characteristics of subjects who complete the study (per protocol analyses). 
Citation: Maggio ABR, Mugnier M, Piaget L, Bessat-Macchi M, Martin X, et al. (2017) Evaluation of a Family-Based Group Therapy for Young Children with Obesity: A Pilot Study. J Family Med Prim Care Open Acc: JFOA-102. DOI: 10.29011/JFOA-102. 100002

\begin{tabular}{|c|c|c|}
\hline & $\frac{\text { Mothers }}{\mathrm{N}=13}$ & $\frac{\text { Fathers }}{\mathrm{N}=13}$ \\
\hline & N (\%) & N (\%) \\
\hline \multicolumn{3}{|c|}{ BDI : } \\
\hline No depression & $5 / 12(41.7)$ & $5 / 8(62.5)$ \\
\hline Mild depression & $1 / 12(8.3)$ & $1 / 8(12.5)$ \\
\hline Moderate depression & $6 / 12(50)$ & $1 / 8(12.5)$ \\
\hline Severe depression & 0 & $1 / 8(12.5)$ \\
\hline Missing questionnaire & $1 / 13(7.7)$ & $5 / 13(38.5)$ \\
\hline \multicolumn{3}{|c|}{ BAI: } \\
\hline No to mild anxiety & $10 / 12(83.3)$ & $5 / 5(100)$ \\
\hline Moderate anxiety & 2/12 (16.7) & 0 \\
\hline Missing questionnaire & $1 / 13(7.7)$ & $8 / 13(61.5)$ \\
\hline \multicolumn{3}{|c|}{ Mixed: } \\
\hline Anxio-depressive & $2(16.7)$ & 0 \\
\hline \multicolumn{3}{|c|}{$\begin{array}{c}\text { Abbreviations: BDI: Beck Depression Inventory; BAI: Beck anxiety } \\
\text { Inventory }\end{array}$} \\
\hline
\end{tabular}

Table 2: Results of BDI and BAI Questionnaires at baseline (per protocol analyses).

Compared to the SDQ normative data, only $30 \%$ of children were considered as normal for total difficulty scoring $(30 \%$ borderline and $40 \%$ abnormal) the father's depression and anxiety levels (BDI and BAI total scores) were positively correlated with the BMI z-score change at 6 months (BDI: $r=0.821, \mathrm{p}=0.012$; BAI: $\mathrm{r}=0.723, \mathrm{p}=0.043$ ) and at 12 months (BDI: $\mathrm{r}=0.793, \mathrm{p}=0.019$; BAI: $\mathrm{r}=0.931, \mathrm{p}=0.001)$. Moreover, maternal and paternal questionnaires completion rates at 12 months were significantly inversely correlated to the BMI z-score change at 12 months (mothers: $\mathrm{r}=-$ $0.608, \mathrm{p}=0.027$; fathers: $\mathrm{r}=-0.698, \mathrm{p}=0.008$ ).

The child's emotional problem score was positively correlated at 12 months with BMI $\mathrm{z}$-score change $(\mathrm{r}=0.606, \mathrm{p}=0.048)$. There was a significant difference between emotionally normal and abnormal subjects (Delta BMI z-score $-0.19 \pm 0.2 \mathrm{~kg} . \mathrm{m}-2$ vs. $0.37 \pm 0.4$ kg.m-2; $\mathrm{p}=0.039)$.

Baseline subjects' characteristics, questionnaires result, and evolution were similar between the high ( $>75 \%$ of sessions) and low participation rate groups (Table 1). However, the paternal questionnaire completion rate at baseline tended to be greater in the high participation rate group (High: 6/7 vs. Low: $2 / 6 ; \mathrm{p}=0.053$ ).

\section{Discussion}

Childhood obesity is a major public health challenge as its prevalence is increasing worldwide and it is tracking into adult- hood, with a 4-fold increased risk for young children aged 2 to- 5 years [5]. This pilot study showed that the BMI z-score was stable during the first 6 months of intervention, but increased after one year, which is in accordance with a systematic review on the same age group [6]. However, the last Cochrane review showed better results but the overall quality of the trials was low [7]. These findings highlight the difficulty for families to sustain efforts over a long period of time.

Surprisingly, the weight outcome was not influenced by the participation rate but rather by the global implication of the family [8] and the psychological states of the child and their father. Indeed, BMI z-score changes were positive when the father was suffering from depressive disorder, and/or when the child was suffering of emotional problems. Paternal depression has been shown to have an impact on adverse emotional and behavioral outcomes in children aged 3 to 5 years [9].

Furthermore, we observed that the participation rate was dependent of the father's support, assessed indirectly through their willingness to complete the questionnaires. This finding suggests that an active implication of the father in the therapeutic process may help the mother and child to sustain their efforts. However, it has to be confirmed in randomized controlled trials comprising the father's participation. A recent review investigated the factors influencing the drop-out rate but surprisingly no study investigated the role of the father [10].

In conclusion, despite a small sample size, this study suggests that it feasible to implement a low-intensity physical activity and FBBT for the weight management of young children, however it is very difficult to reduced or maintain the BMI z-score over time. This study highlights the influence of the child's behavior and of the father's support and psychological states for the participation in a weight management program.

\section{Compliance with Ethical Standards}

Conflict of interest: The authors have no conflicts of interest to declare.

Funding: This study was supported financially by the Geneva Pediatric Network, the Hans Wilsdorf foundation and the University Hospitals of Geneva. The work was independent of the funding.

Informed consent : The Ethics Commission for Research on the Human Being (CEREH) ( $n^{\circ}$ de ref. CER :13-172) ethics committee approved this study and a written informed consent was obtained from parents.

\section{Acknowledgements}

We thank the subjects for volunteering for the study and the Department of the Public Instruction of the Geneva state for the free utilization of the premises. We thank the "Quarantezéro ten- 
Citation: Maggio ABR, Mugnier M, Piaget L, Bessat-Macchi M, Martin X, et al. (2017) Evaluation of a Family-Based Group Therapy for Young Children with Obesity: A Pilot Study. J Family Med Prim Care Open Acc: JFOA-102. DOI: 10.29011/JFOA-102. 100002

nis academy" and Murielle Thurnherr, psychomotor therapist, for their participation.

\section{Authors' contribution:}

MM, LP, and MBM conceived and carried out experiments, VD, NFL, AR and JSL conceived experiments. AM conceived experiments, analyzed data and wrote the manuscript. XM carried out experiments. All authors were involved in writing the paper and had final approval of the submitted and published versions.

\section{References}

1. Epstein LH, Valoski A, Koeske R, Wing RR (1986) Family-based behavioral weight control in obese young children. J Am Diet Assoc 86: 481-484.

2. Goodman R (1997) The Strengths and Difficulties Questionnaire: a research note. J Child Psychol Psychiatry 38: 581-586.

3. Beck AT, Ward CH, Mendelson M, Mock J, Erbaugh J (1961) An inventory for measuring depression. Arch Gen Psychiatry 4: 561-571.

4. Beck AT, Epstein N, Brown G, Steer RA (1988) An inventory for measuring clinical anxiety: psychometric properties. J Consult Clin Psychol 56: 893-897.
5. Freedman DS, Khan LK, Serdula MK, Dietz WH, Srinivasan SR, et al. (2005) The relation of childhood BMI to adult adiposity: the Bogalusa Heart Study. Pediatrics 115: 22-27.

6. Bond M, Wyatt K, Lloyd J, Taylor R (2011) Systematic review of the effectiveness of weight management schemes for the under fives. Obes Rev 12: 242-253.

7. Colquitt JL, Loveman E, O'Malley C, Azevedo LB, Mead E, et al. (2016) Diet, physical activity, and behavioural interventions for the treatment of overweight or obesity in preschool children up to the age of 6 years. Cochrane Database Syst Rev 3: CD012105.

8. Bracale R, Milani Marin LE, Russo V, Zavarrone E, Ferrara E, et al. (2015) Family lifestyle and childhood obesity in an urban city of Northern Italy. Eat Weight Disord 20: 363-370.

9. Ramchandani P, Stein A, Evans J, O'Connor TG (2005) Paternal depression in the postnatal period and child development: a prospective population study. Lancet 365: 2201-2205

10. Skelton JA, Beech BM (2011) Attrition in paediatric weight management: a review of the literature and new directions. Obes Rev 12 e273-e281. 